Table 1. Clinical Features of Patiens

\begin{tabular}{|c|c|c|c|c|c|c|c|}
\hline & 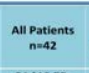 & $\begin{array}{c}\text { ESC/ESR } \\
\text { screen } \\
\text { positive, n=7 } \\
\text { (17\%) }\end{array}$ & $\begin{array}{c}\text { ESC/ESR } \\
\text { screen } \\
\text { negative, } \\
\text { nezs (83\%) }\end{array}$ & $\begin{array}{c}\text { DEFrect } \\
\text { screen } \\
\text { positive, n=13 } \\
\text { (31x) }\end{array}$ & $\begin{array}{c}\text { DETECT } \\
\text { screen } \\
\text { negative, n= } \\
29(69 \%)\end{array}$ & 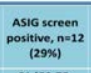 & $\begin{array}{l}\text { ASIG screen } \\
\text { negative, } \\
n=19(59 \%)\end{array}$ \\
\hline Age, median [IQR)] & $\begin{array}{l}51 / 46,75 \\
5975\end{array}$ & 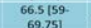 & $48[38.75-52]$ & $\begin{array}{c}66.5158 .25 . \\
6975\end{array}$ & 48 [38.5-51.5] & 6152.75 & 48 [38.51] \\
\hline Male/female, $n(x)$ & $3 / 39$ & & 3/32 & $2 / 11$ & $1 / 28$ & $0 / 12$ & 3/27 \\
\hline Duration of SSc symptoms, vear [lQR] & $13.5[5-20.75]$ & $52[39.57]$ & 65 [51-79.5] & 24 [5-38] & $\begin{array}{c}\begin{array}{c}125.25- \\
16.751\end{array} \\
\end{array}$ & $14[5-37]$ & $13|5-19|$ \\
\hline $\begin{array}{l}\text { Diffuse } \\
\text { Uimited }\end{array}$ & $\begin{array}{l}\begin{array}{l}12(28) \\
30(72)\end{array} \\
\text { (1) }\end{array}$ & $\begin{array}{l}2(29) \\
5(71)\end{array}$ & $\begin{array}{l}10(29) \\
25(71)\end{array}$ & $\begin{array}{l}2(15) \\
11(85)\end{array}$ & $\begin{array}{l}101341 \\
19 /(66)\end{array}$ & $\begin{array}{l}4(67) \\
8(67)\end{array}$ & $\begin{array}{l}8(27) \\
22(73)\end{array}$ \\
\hline Anti-Scl70 $(+)$ & 20/33(61) & $3 / 6$ (50) & $17 / 27(63)$ & $5 / 11(44)$ & $15 / 22(68)$ & $5 / 9(56)$ & $15 / 24(62.5)$ \\
\hline $\begin{array}{l}\text { Anticentromere }(+) \\
\text { of } 1 \mathrm{D}, n(x)\end{array}$ & $8 / 39(21)$ & $\begin{array}{c}3 / 7(43) \\
4(157)\end{array}$ & $\begin{array}{l}5 / 32(16) \\
21(60)\end{array}$ & $\begin{array}{l}6 / 12(50) \\
9(69)\end{array}$ & $\begin{array}{l}2 / 27(7) \\
16(55)\end{array}$ & $\begin{array}{l}4433) \\
7(38)\end{array}$ & $4 / 27(15)$ \\
\hline 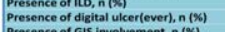 & 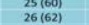 & $\begin{array}{l}4(57) \\
3(43)\end{array}$ & & $\begin{array}{l}9(69) \\
8(62)\end{array}-2$ & & $\begin{array}{l}7758) \\
8(67)-30\end{array}$ & $\begin{array}{l}18(60) \\
18(62)\end{array}$ \\
\hline 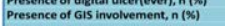 & $20(48)$ & 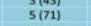 & $15(43)$ & $\begin{array}{l}8(162) \\
6(52)\end{array}$ & $\begin{array}{l}1450) \\
14(50)\end{array}$ & $6 / 11(55)$ & $\begin{array}{l}18(62) \\
14(50)\end{array}$ \\
\hline Presence of telangiectasia, $n$ (\%) & 23 (55) & $5(71)$ & $18(58)$ & $9(75)$ & $14(54)$ & $8 / 10(80)$ & $15 / 28(54)$ \\
\hline sPAP on TTE, median (MmHE) [IQR] & 30 [26-34] & $41[34-46]$ & 28 [26-32] & 32 [30-43] & 28 [26-34] & $38[30.42]$ & 28 126-32] \\
\hline FVC, median (X predicted) [IQR] & $\begin{array}{l}87.5171 .5 \\
100.251\end{array}$ & $94[62-11]]$ & $86[72.99]$ & $82[71 \cdot 96]$ & 79 [69.25-100] & $\begin{array}{l}94 \mid 69.25- \\
115.25]\end{array}$ & $\begin{array}{l}84.5[71.5 . \\
97.5]\end{array}$ \\
\hline DLCO, median (\$ predicted) (IIQR] & $\begin{array}{l}60.50150 .75- \\
72.51\end{array}$ & $52[39.57)$ & $64[51.78]$ & $57[43.5 .73 .5]$ & $61 \mid 51.76]$ & 53 [43.59.25] & $\begin{array}{l}65[51.75 \cdot \\
78.51\end{array}$ \\
\hline FVC / Dico ratio, median [IQR] ] & $\begin{array}{l}1.39[1.18 . \\
1.72]\end{array}$ & $\begin{array}{l}2.11[1.58 . \\
2.18\}\end{array}$ & $\begin{array}{c}1.35 \mid 1.16- \\
1.55 \mid\end{array}$ & $\begin{array}{c}1.64[1.29 . \\
2.15]\end{array}$ & $\begin{array}{c}1.36[1.13- \\
1.57]\end{array}$ & $\begin{array}{c}1.84[1.41 . \\
2.12]\end{array}$ & $1.35[1.0-1.47]$ \\
\hline NT-proBNP, median (PE/mL) (IIRR] & $\begin{array}{c}141(62.75 \\
196]\end{array}$ & $310[210-887]$ & $120[52-168]$ & $\begin{array}{c}4210[133.5 \\
546]\end{array}$ & $120[45 \cdot 163.5]$ & $\begin{array}{c}260[149 . \\
593.51\end{array}$ & $\begin{array}{c}114.5[48.5 . \\
165.75]\end{array}$ \\
\hline $\begin{array}{l}\text { Presence of dvspnea, } n(x) \\
\text { Class } 1\end{array}$ & $16(38)$. & $7(100)$ & $9(26)$ & $6(46)$ & $10(35)$ & $7(58)$ & $9(30)$ \\
\hline $\begin{array}{l}\text { class } 2 \\
\text { class } 3\end{array}$ & $\begin{array}{c}14(93) \\
1(7)\end{array}$ & $\begin{array}{l}6(86) \\
1(14)\end{array}$ & $8(100)$ & $\begin{array}{l}5(83) \\
1(17)\end{array}$ & $9 / 9(100)$ & $\begin{array}{l}6(86) \\
1(14)\end{array}$ & $8(100)$ \\
\hline Class 4 & & & 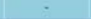 & & 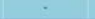 & & $\cdot$ \\
\hline
\end{tabular}

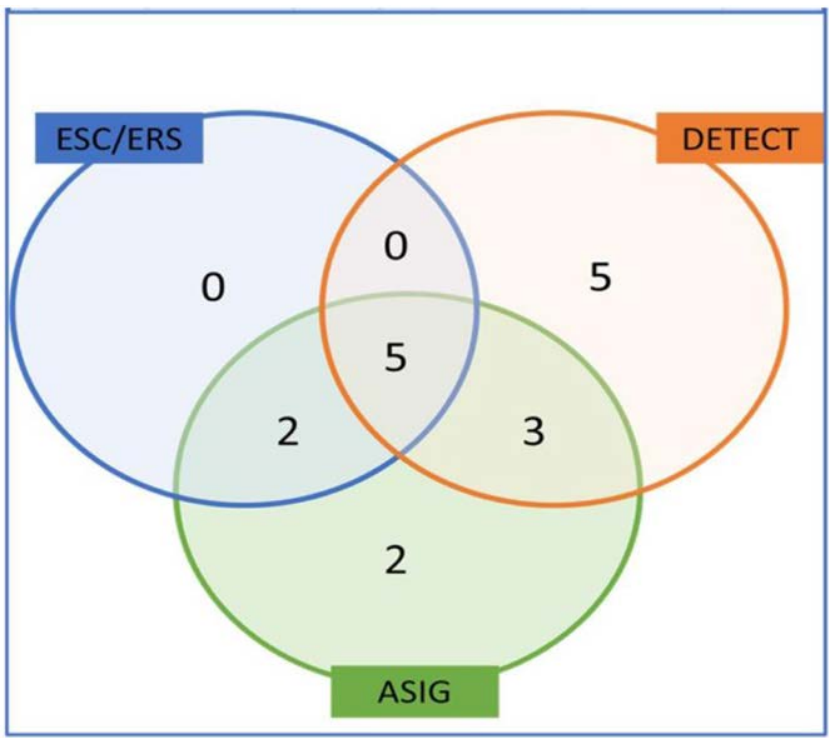

Figure. Number of patients who required RHC according to the algorithms

\begin{tabular}{|c|c|c|c|c|c|c|}
\hline & \multicolumn{3}{|c|}{$\underline{25 \mathrm{mmHg} \text { cut-off }}$} & \multicolumn{3}{|c|}{$20 \mathrm{mmHg}$ cut-off } \\
\hline & ESC/ESR & ASIG & DETECT & ESC/ESR & ASIG & DETECT \\
\hline Sensitivity, \% & 67 & 100 & 100 & 56 & 89 & 56 \\
\hline Specifity, \% & 64 & 36 & 29 & 75 & 50 & 0 \\
\hline PPV, \% & 29 & 25 & 23 & 71 & 67 & 39 \\
\hline NPV,\% & 90 & 100 & 100 & 60 & 80 & 0 \\
\hline
\end{tabular}

Disclosure of Interests: Mustafa Erdogan: None declared, Burcak Kilickiran Avci: None declared, Cansu Ebren: None declared, Yagmur Ersoy: None declared, Zeki Ongen: None declared, Gul Ongen: None declared, Vedat Hamuryudan Speakers bureau: Pfizer, AbbVie, Amgen, MSD, Novartis, UCB, Gulen Hatemi Grant/research support from: BMS, Celgene Corporation, Silk Road Therapeutics - grant/research support, Consultant of: Bayer, Eli Lilly - consultant, Speakers bureau: AbbVie, Mustafa Nevzat, Novartis, UCB - speaker DOI: 10.1136/annrheumdis-2020-eular.3826

\section{FRI0238 \\ AUTOLOGOUS FAT GRAFTING IN THE TREATMENT OF FACIAL SCLERODERMA: A SINGLE - CENTRE EXPERIENCE}

P. Faggioli ${ }^{1}$, L. Castelnovo ${ }^{1}$, A. Tamburello ${ }^{1}$, M. Falaschi ${ }^{1}$, E. Saporiti ${ }^{1}$, A. M. Lurati ${ }^{2}$, A. Laria ${ }^{2}$, A. Mazzone ${ }^{1} .{ }^{1}$ Ospedale di Legnano, Legnano, Italy;

${ }^{2}$ Ospedale Giuseppe Fornaroli, Magenta, Italy
Background: Systemic sclerosis (SSc) is a systemic autoimmune disease characterized by progressive cutaneous and internal organ fibrosis. Orofacial manifestations are disabling and treatment options are limited. Fat Tissue Grafting (FTG) can be used for treating facial manifestations of the fibrosis.

Objectives: In this study, we aimed to assess the safety and efficacy of FGT of our cohort of patients with SSc.

Methods: We enrolled 20 SSc (18W, 2 M) patients, from 2016 to 2019, suffering from facial sclerosis and restricted mouth opening capacity. FTG was carried out in accord with modified Colemans' procedure (1): fat tissue was taken from periumbilical or trochanteric areas and was injected in 8 different points around the mouth. No side effects or adverse reactions have been documented. Evalu ations included mouth opening capacity by measuring interincisal distance, oral functionality (MHISS scale) and patient global satisfaction (by Global Health scale).

Results: A $11 \mathrm{~mm}$ ( 8 - 18mm range) median increase of interincisal distance was reported at month 6 and in $80 \%$ of patients at month 12 , too $(p<0.03)$. A significant improvement in MHISS scale was also observed $(p<003)$. The patient satisfaction questionnaire showed $95 \%$ positive results and $80 \%$ of the patient replied affirmatively to the question about the repetition of FTG but only 2 patients required new FTG after 12 months.

Conclusion: Our results showed that FTG improved mouth opening capacity and that aesthetic and functional results were satisfying to about $90 \%$ of the patients; long-term effects of this type of treatment are currently unknown. However, our and litterature data at 12 months follow-up seems to confirm the benefits in long term, despite the filling effect is over

This study - that's one of the largest case series described right now (2) - supports the possible therapeutic role of autologous FTG in improving facial scleroderma both in aesthetic and in functional aspects.

\section{References:}

[1] Coleman SR. Structural fat grafting: more than a permanent filler. Plast Reconstr Surg. 2006; 118: 108S-20S

[2] Fat Grafting for the Treatment of Scleroderma.Strong AL, Rubin JP, Kozlow JH, Cederna PS. Plast Reconstr Surg. 2019 Dec;144(6):1498-1507. doi: 10.1097/PRS.0000000000006291.

Disclosure of Interests: None declared

DOI: 10.1136/annrheumdis-2020-eular.1527

\section{FRI0239 \\ ANTI-NXP2 ANTIBODIES: CLINICAL AND SEROLOGICAL ASSOCIATIONS IN A MULTICENTRIC} ITALIAN STUDY

M. Fredi ${ }^{1}$, I. Cavazzana ${ }^{1}$, A. Ceribelli ${ }^{2}$, M. G. Lazzaroni ${ }^{1}$, S. Barsotti ${ }^{3}$, M. Benucci ${ }^{4}$, L. Cavagna ${ }^{5}$, L. De Stefano ${ }^{5}$, A. Doria ${ }^{6}$, G. Emmi $^{7}$, M. Fornaro ${ }^{8}$, F. Furini ${ }^{9}$, R. Gerli ${ }^{10}$, M. G. Giudizi ${ }^{7}$, M. Govoni $^{9}$, A. Ghirardello ${ }^{6}$, L. laccarino ${ }^{6}$, F. lannone ${ }^{8}$, M. Infantino ${ }^{4}$, A. Mathieu ${ }^{11}$, E. Marasco ${ }^{5}$, P. Migliorini ${ }^{12}$, B. Palterer $^{7}$ P. Parronchi ${ }^{7}$, M. Piga ${ }^{11}$, F. Pratesi ${ }^{12}$, A. Radice ${ }^{13}$, C. Selmi ${ }^{2}$, V. Riccieri ${ }^{14}$, M. Tampoia ${ }^{15}$, G. Zanframundo ${ }^{5}$, A. Tincani ${ }^{1}$, F. Franceschini ${ }^{1} .{ }^{1}$ Rheumatology Unit Spedali Civili Università di Brescia, Brescia, Italy; ${ }^{2}$ Rheumatology Unit Humanitas, Rozzano, Italy; ${ }^{3}$ Rheumatology Unit AOU Pisana, Pisa, Italy; ${ }^{4}$ Rheumatology Unit Ospedale S. Giovanni di Dio, Firenze, Italy; ${ }^{5}$ Rheumatology Unit Policlinico San Matteo, Pavia, Italy; ${ }^{6}$ Rheumatology Unit Università Studi di Padova, Padova, Italy; ${ }^{7} \mathrm{AO}$ Universitaria Careggi, Firenze, Italy; ${ }^{8}$ Rheumatology Unit Università di Bari, Bari, Italy; ${ }^{9}$ Rheumatology Unit Università di Ferrara, Ferrara, Italy; ${ }^{10}$ Rheumatology Unit Università di Perugia, Perugia, Italy;

${ }^{11}$ Rheumatology Unit, Università di Cagliari, Cagliari, Italy; ${ }^{12}$ Immunoallergologia, AO Universitaria Pisana, Pisa, Italy; ${ }^{13}$ Microbiologia e Virologia Ospedale San Carlo Borromeo, Milano, Italy; ${ }^{14}$ Policlinico Umberto I, Roma, Italy; ${ }^{15}$ Laboratorio Patologia Clinica Policlinico Bari, Bari, Italy

Background: anti-NXP2 antibodies is considered a serological marker of dermatomyositis (DM), with calcinosis, severe myositis and, in some series, cancer Historically, these associations have been detected with immunoprecipitation (IP), but in the last few years commercial lineblot (LB) assay have been released. Objectives: to analyze the clinical features associated to anti-NXP2 antibodies including the onset of concomitant cancers, both with LB and homemade IP Methods: clinical and serological data from medical charts of 213 patients with a diagnosis of inflammatory miosidites without anti-NXP2 (NXP2-), followed-up by two third-level Centers, and 61 anti-NXP2+ patients from 10 Rheumatological centers were analyzed. Anti-myositis specific (MSA) and anti-myositis associated antibodies (MAA) were detected in single centers by LB (Euroimmun Autoimmune Inflammatory Myopathies 16 antigens). Anti-NXP2 was confirmed by protein and RNA IP, as previously described (1)

Results: clinical diagnosis of anti-NXP2+ positive with LB were $42 \mathrm{DM}, 11 \mathrm{PM}$, inclusion body myositis (IBM) 4, necrotizing myositis and overlap (OM) 1 each Anti-NXP2 + showed a lower age at onset $(\mathrm{p}<0.0001)$ more frequent diagnosis of DM (68.8\%vs30\%,OR5.2) and IBM (6.5\%vs0.49\%,OR14.8), typical skin manifestations, myositis (93\%vs79\% OR3.3), concomitant presence of another MSA (12.7\%vs2\%, OR6.41) and lower rate of features associated with OM or anti-synthetase syndrome. Serum from 49 NXP2+ was available and IP analysis 